\title{
Promyelocytic leukemia antigen expression: A histological marker for primary biliary cholangitis diagnosis?
}

\author{
Panagiotis A. Papamichalis ${ }^{1,2}$, Kalliopi Zachou ${ }^{2}$, Roidoula A. Papamichali ${ }^{1}$, \\ Maria Ioannou $^{1}$, Nikolaos K. Gatselis ${ }^{2}$, George N. Dalekos ${ }^{2}$, George K. Koukoulis ${ }^{1}$ \\ 'Department of Pathology, Medical School, University of Thessaly, 41110 Larissa, Greece; \\ 2Department of Medicine and Research Laboratory of Internal Medicine, National Expertise Center of Greece in \\ Autoimmune Liver Diseases, General University Hospital of Larissa, 41110 Larissa, Greece
}

\section{ABSTRACT}

Background and Objectives: Distinguishing primary biliary cholangitis (PBC) from other cholestatic diseases at the histological level could be assisted by new methods, such as immunohistochemical staining of specific antigens. Methods: We evaluated whether the detection of promyelocytic leukemia protein (PML) can serve as a specific and sensitive marker for PBC diagnosis. Liver biopsies from 26 PBC patients, 20 primary sclerosing cholangitis (PSC), 37 viral hepatitis, 11 non-alcoholic steatohepatitis (NASH) and 5 normal patients were investigated after immunostaining with the anti-PML monoclonal PG-M3, IgG1 antibody. Results: Immunoreactivity in bile ducts was expressed by the PML-score (quotient of positive ducts to the total number of portal tracts multiplied by 2). PML-score was higher in PBC as compared to controls $(P<0.001)$. Using a cutoff of 0.18 , PML-score proved highly sensitive $(84.6 \%)$ and specific $(89.7 \%)$ for confirming PBC as compared to only $5 \%$ of PSC, $9.1 \%$ of $\mathrm{NASH}$ and $13.5 \%$ of viral hepatitis patients $(P<0.001)$. Irrespective of the underlying disease, patients with PML-score $>0.18$ were older $(P=0.007)$, more often females $(P<0.001)$ with higher ALP $(P<0.001)$, y-GT $(P=0.001)$ and $\operatorname{lgM}(P<0.001)$ compared to the patients with PML-score $<0.18$. Conclusions: We postulate that a simple PML immunohistochemical test could be sufficient for histopathological discrimination of $\mathrm{PBC}$ in problematic cases of undefined cholestatic disorders, including small-duct PSC and AMA-negative PBC cases.

Key words: primary biliary cholangitis, primary sclerosing cholangitis, anti-nuclear antibodies, anti-PML antibodies, immunohistochemical staining

Address for Correspondence: Dr. George N. Dalekos, MD, PhD, Professor of Medicine, Head of the Department of Medicine and the Research Laboratory of Internal Medicine, National Expertise Center of Greece in Autoimmune Liver Diseases, General University Hospital of Larissa, Larissa 41110, Greece.

E-mail: dalekos@med.uth.gr

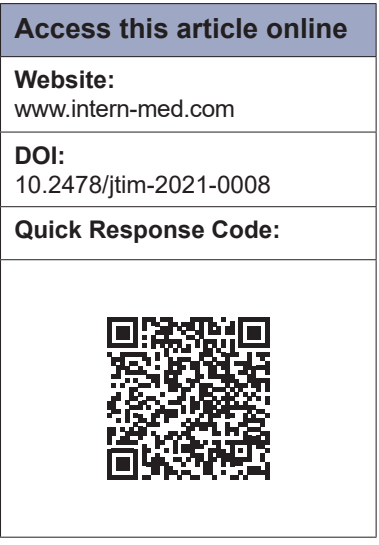

\section{INTRODUCTION}

Primary biliary cholangitis (PBC) is an autoimmune cholestatic liver disease that primarily affects middle-aged women, characterized by progressive destruction and loss of small intrahepatic bile ducts, progressive fibrosis and the eventual development of cirrhosis and liver failure. ${ }^{[1,2]}$ Anti-mitochondrial antibodies (AMA) are considered the laboratory hallmark of the disease, being detected in $90 \%-95 \%$ of patients. ${ }^{[3,4]}$ Additionally, more than 60 autoantibodies have been explored in PBC patients including anti-nuclear antibodies (ANA). ${ }^{[3-7]}$ However, none of them has been proven to associate strongly with disease pathogenesis.
ANA in PBC mainly involve antibodies against many autoantigens including sp-100 (anti-sp100), gp210 (anti-gp210), lamin B receptor (anti-LBR), promyelocytic leukemia (anti-PML), p62 (anti-p62), sp140, centromere, Kelch-like 12 and hexokinase-1. ${ }^{[8-10]}$ Using indirect immunofluorescence (IIFL) on HEp2 cells, they produce distinct patterns: homogeneous, multiple nuclear $\operatorname{dot}(\mathrm{MND})$, speckled, centromere, or rimlike/membranous (RLM) $\cdot^{[3-5]}$ ANA patterns highly specific for $\mathrm{PBC}$ diagnosis include the RLM-pattern (characteristic for anti-gp210, anti-LBR, anti-p62 presence) and the MNDpattern (characteristic for anti-sp100 and/ or anti-PML presence). ${ }^{[9,11]}$ Anti-PML and anti-sp100 antibodies, which co-localize in intra-nuclear structures called PML-nuclear 
bodies (PML-NBs), are usually detected simultaneously in the sera of patients ${ }^{[5,8,12]}$ being induced by interferons. ${ }^{[13]}$

Liver biopsy is still needed in cases negative for the PBCspecific antibodies, or when concurrent autoimmune hepatitis (AIH) or other variant forms or non-alcoholic steatohepatitis (NASH) is suspected or when there is presence of other extrahepatic comorbidities. ${ }^{[14,15]} \mathrm{PBC}$ staging is based on the degree of bile duct damage, inflammation and fibrosis. ${ }^{[16,17]}$ Focal duct obliteration with granuloma is almost pathognomonic. However, the lesions may have heterogeneous distribution and histological features characterizing different stages can be seen in the same liver biopsy specimen. ${ }^{[18-20]}$

An old, yet still standing diagnostic challenge is the histological discrimination of diseases presenting with lesions under the terminology of non-suppurative cholangitis. Granulomatous, lymphoid, fibrous (fibrousobliterative) and pleomorphic cholangitis are the four subtypes of non-suppurative cholangitis. ${ }^{[21]}$ Apart from $\mathrm{PBC}$, primary sclerosing cholangitis (PSC) and viral hepatitis, especially hepatitis $\mathrm{C}$ virus (HCV), may present with some forms of non-suppurative cholangitis that have to be distinguished from PBC. The distinction could be even more difficult between PBC and PSC as these diseases not only share some common clinical features but also histological ones. New diagnostic approaches and methods, like immunohistochemistry on liver tissues could help towards the morphologic distinction of the diseases.

Under this context, the presence of specific antigens could be helpful not only for disease characterization and differential diagnosis, but also for significant pathogenetic implications; therefore, in this preliminary study, we evaluated the specificity, sensitivity and diagnostic accuracy along with the potential clinical significance of PML detection in the nuclei of bile ducts in PBC biopsies as similar data on this issue is missing. Patients with PSC, viral hepatitis and NASH were also investigated consisting the disease control group.

\section{MATERIALS AND METHODS}

\section{Subjects}

Twenty-six consecutive patients fulfilling all three diagnostic criteria for PBC (elevated cholestatic enzymes, liver histology and AMA positivity [titre $\geq 1: 40])^{[1-4]}$ were included in the study. Within the same period, we investigated 20 PSC, 11 NASH and 37 patients with viral hepatitis (25 with chronic hepatitis B virus [HBV] and 12 with chronic HCV infections) who served as the disease control groups while 5 liver specimens from healthy subjects obtained during routine cholecystectomy served as healthy controls. Healthy subjects had apparently no evidence of chronic liver disease and normal alanine aminotransferase (ALT) values, tested negative for HBV and HCV and denied ever having used hepatotoxic drugs, herbals, or having abused alcohol or injected drugs. The demographic, clinical, laboratory and histological characteristics of patients included in the study are shown in Table 1. Diagnosis of PSC, NASH, HBV and HCV was based on recent clinical practice guidelines. ${ }^{[22-26]}$

Aspartate aminotransferase (AST), ALT, alkaline phosphatase (ALP), gamma-glutamyltranspeptidase $(\gamma-G T)$, total bilirubin (TBil), albumin and IgM immunoglobulin were determined using standard techniques. The Mayorisk score, GLOBE-score and Paris II criteria of response to treatment were calculated in PBC patients. ${ }^{[27-29]}$ The ethical committee of the General University Hospital of Larissa approved the study protocol, which conforms to the ethical guidelines of the 1975 Declaration of Helsinki as reflected in a priori approval by the institution's human research committee.

\section{Immunohistochemical detection of PML in bile ducts}

Formalin-fixed, paraffin-embedded liver tissues were sectioned at 3 to $5 \mu \mathrm{m}$ thickness, mounted on silanecoated slides and dried overnight at $37^{\circ} \mathrm{C}$. The sections were then dewaxed, by sequential immersion in xylene, graded concentrations of ethanol, and distilled water. Antigen retrieval was performed by heating the slides in covered glass Coplin jars with $0.01 \mathrm{~mol} / \mathrm{L}$ sodium citrate buffer (pH 6.0), three times for 5 minutes, at $700 \mathrm{~W}$, in a microwave oven. The sections were then allowed to cool down at room temperature for approximately 15 minutes, in Tris-buffered saline with Tween (TBST), and subjected to immunostaining procedure, as described below.

Sections were placed in 3\% hydrogen peroxide solution for 10 minutes and then washed with TBST, followed by the incubation of the slides with the primary antibody for 30 minutes. PML protein was labelled with the monoclonal mouse anti-Human PML Protein (DAKO), clone: PGM3, isotype IgG1, kappa, in 1:50 dilution. The sections were then washed with TBST and Envision polymer (EnVision'TM Detection system, K5007, Dako) was applied for 30 minutes. The sections were washed with TBST and incubated with 3,3'- diaminobenzidine for 5 minutes. The sections were washed again with TBST and counterstained for 3 minutes in 10\% Harris hematoxylin. Then, the sections were rinsed with running tap water and distilled water. Thereafter, the sections were dehydrated with graded ethanol and xylenes and cover slipped. Negative controls consisted of substitution of primary antibody with preimmune serum or application of chromogen alone. 
Papamichalis et al.: Promyelocytic leukemia antigen expression: A histological marker for primary biliary cholangitis diagnosis?

\begin{tabular}{|c|c|c|c|c|}
\hline & $\begin{array}{l}\text { PBC } \\
(n=26)\end{array}$ & $\begin{array}{l}\text { PSC } \\
(n=20)\end{array}$ & $\begin{array}{l}\text { NASH } \\
(n=11)\end{array}$ & $\begin{array}{l}\text { Viral hepatitis } \\
(n=37)\end{array}$ \\
\hline Age (years) & $53(32-73)$ & $41(27-72)$ & $44(26-69)$ & $49(16-76)$ \\
\hline Sex (male/female) & $2 / 24$ & $14 / 6$ & $8 / 3$ & $26 / 11$ \\
\hline AST (U/L; ULN: 40) & $37.5(12-204)$ & $34(19-150)$ & $34(21-1286)$ & $41(16-449)$ \\
\hline ALT (U/L; ULN: 40) & $38(9-324)$ & $55.5(11-278)$ & $57(12-1251)$ & $50(9-657)$ \\
\hline ALP (U/L; ULN: 104) & $138(80-606)$ & $101(50-677)$ & $79(40-647)$ & $65(34-180)$ \\
\hline$\gamma$-GT (U/L; ULN: 40) & $135(22-883)$ & $84(11-874)$ & $25(12-184)$ & $35(7-232)$ \\
\hline TBil (mg/dl; ULN: 1) & $0.6(0.35-1.6)$ & $0.8(0.4-4.5)$ & $0.5(0.4-4.8)$ & $0.7(0.35-2.6)$ \\
\hline Albumin $(\mathrm{g} / \mathrm{dL})$ & $4.3(3.18-5)$ & $4.3(3.3-5.5)$ & $4.4(3.9-4.8)$ & $4.2(3.4-4.9)$ \\
\hline $\lg M(\mathrm{mg} / \mathrm{dL})$ & $300(56-834)$ & $111(37-425)$ & $79(35.2-166)$ & $107(37-324)$ \\
\hline $\begin{array}{l}\text { Cirrhosis (yes/no) } \\
\text { (histology) }\end{array}$ & $1 / 25$ & $1 / 19$ & $0 / 11$ & $8 / 29$ \\
\hline
\end{tabular}

Data are expressed as median (range). ULN: upper limit of normal; PBC: primary biliary cholangitis; PSC: primary sclerosing cholangitis; NASH: non-alcoholic steatohepatitis; AST: aspartate aminotransferase; ALT: alanine aminotransferase; $\gamma$-GT: gamma-glutamyltranspeptidase; ALP: alkaline phosphatse; TBil: total bilirubin.

Quantification of PML protein immunostaining was achieved by the estimation of a PML-score. In each liver biopsy, both the number of portal tracts and the number of interlobular bile ducts (not the small, peripherally placed ductules) showing PML immunostaining were counted by two experienced pathologists (G.K. and R.P.) who were unaware of the clinical diagnosis. The quotient of "positive" bile ducts to the total number of portal tracts was multiplied by 2 , when the intensity of the immunohistochemical detection was so strong that could be seen by the observer with the use of low power objective lens of the microscope. Thus, maximum PML-score value could be 2 , and minimum 0 .

\section{Autoantibody detection in patients' sera}

Presence of AMA and ANA was initially detected by IIFL on $5 \mathrm{~mm}$ fresh frozen sections of in-house rodent multiorgan (kidney, liver and stomach) tissue substrates using, as revealing reagent, a rabbit anti-human Ig-fluorescein isothiocyanate conjugate (FITC; $\operatorname{IgG}, \operatorname{IgA}, \operatorname{IgM}, \mathrm{k}$ and 1; Dako Ltd, Denmark), as we described previously. ${ }^{[30-32]}$ Serum samples were screened at a dilution of $1 / 40$ in accordance with our laboratory practice. AMA reactivity was further evaluated by a commercial ELISA (QUANTA Lite ${ }^{\circledR}$ M2-EP [MIT3] ELISA; INOVA Diagnostics, Inc San Diego, CA $)^{[33]}$ and by Western immunoblot using the mitochondrial fraction of rat liver extracts. ${ }^{[3,4]}$

To better define the ANA pattern, all the sera were also evaluated by IIFL using commercially available HEp-2 cells (NOVA Lite ${ }^{\circledR}$ HEp-2 ANA kit, INOVA Diagnostics). ${ }^{[3,4,11]}$ In addition, all the sera were further tested by a commercial immunoblot assay which uses purified, biochemically characterized antigens (EUROLINE profile autoimmune liver diseases [AMA-M2, M2-3E, Sp100, PML, gp210, LKM-1, LC-1, SLA/LP, Ro-52], EUROIMMUN,
Germany) for anti-PML and anti-sp100 antibodies, according to manufacturer's instructions.

\section{Liver histology}

Histological diagnosis was performed by an experienced hepatopathologist (G.K.) who was unaware of the clinical and laboratory data of the patients. Liver biopsies of PBC patients were assessed according to Ludwig's classification. ${ }^{[14]}$

\section{Statistical analysis}

Results are expressed as median (range) and mean \pm SD, where appropriate. Data were analyzed by two $\chi^{2}$ with Yates' correction test, Fisher's exact test and Mann-Whitney U test. The receiver operating characteristic (ROC) was used for the estimation of the area under the curve (AUC), sensitivity and specificity. A two-sided t-test value of $P<$ 0.05 was considered as statistically significant.

\section{RESULTS}

Representative intense and diffuse nuclear PML immunohistochemical staining of ductal epithelial cells in interlobular bile ducts of PBC cases are shown in Figures 1 and 2 whereas, no immunostaining is seen in a representative PSC case (Figure 3).

PML-score was significantly higher in patients with PBC than in the total number of patients of the disease controls (0.666 [0-2] vs. $0[0-1] ; P=0.001]$. More specifically, PMLscore was higher in $\mathrm{PBC}$ patients than in each disease control group (PSC: 0 [0-0.583], NASH: 0 [0-0.5], viral hepatitis: 0 [0-1], $P<0.0001$ for each comparison) while the PML-score did not differ among PSC, NASH and viral hepatitis patients (Figure 4). 


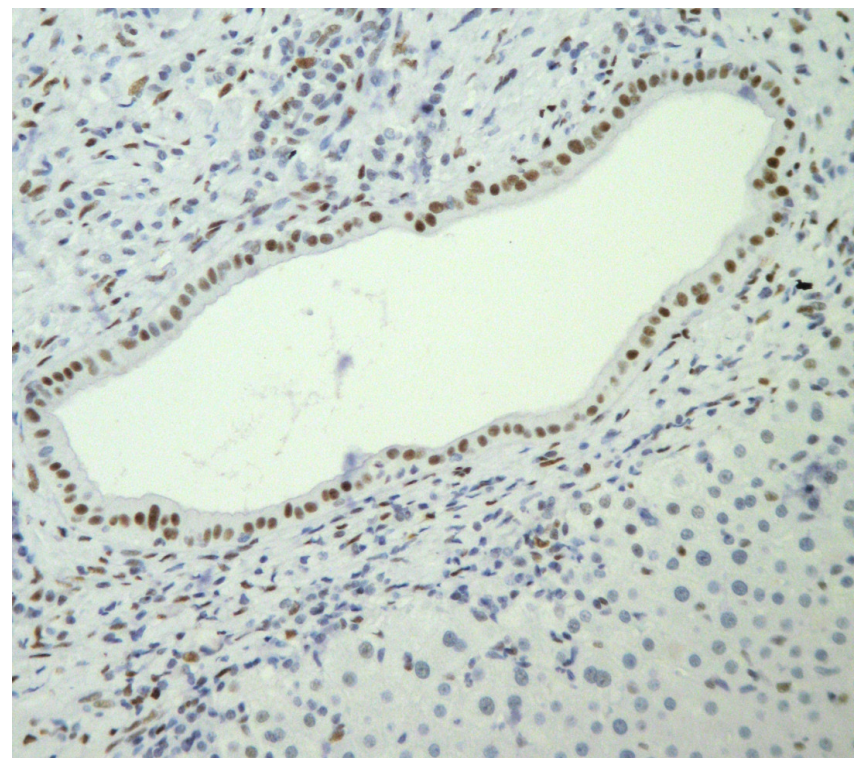

Figure 1: Intense nuclear immunostaining is seen in a bile duct from a biopsy of a PBC patient (Initial magnification $200 \times$ ).

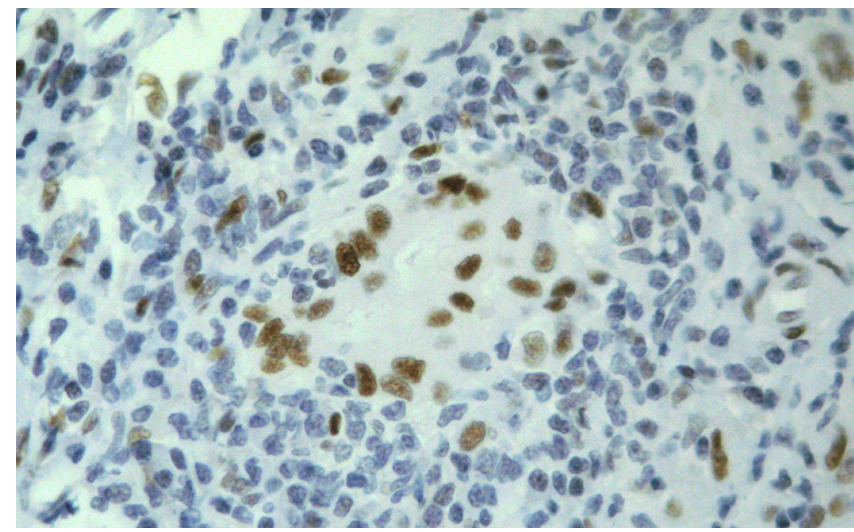

Figure 2: Intense and diffuse nuclear staining of ductal epithelial cells in an interlobular bile duct in a case of PBC (Original magnification $400 \times$ ).

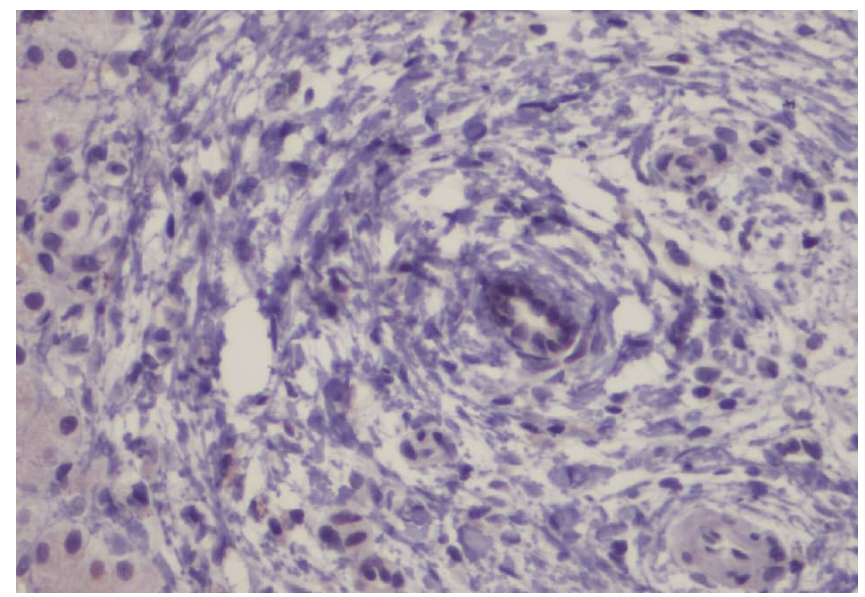

Figure 3: No immunostaining for PML is seen in a case of PSC that included an interlobular bile duct with periductal fibrosis (Original magnification $400 \times$ ).

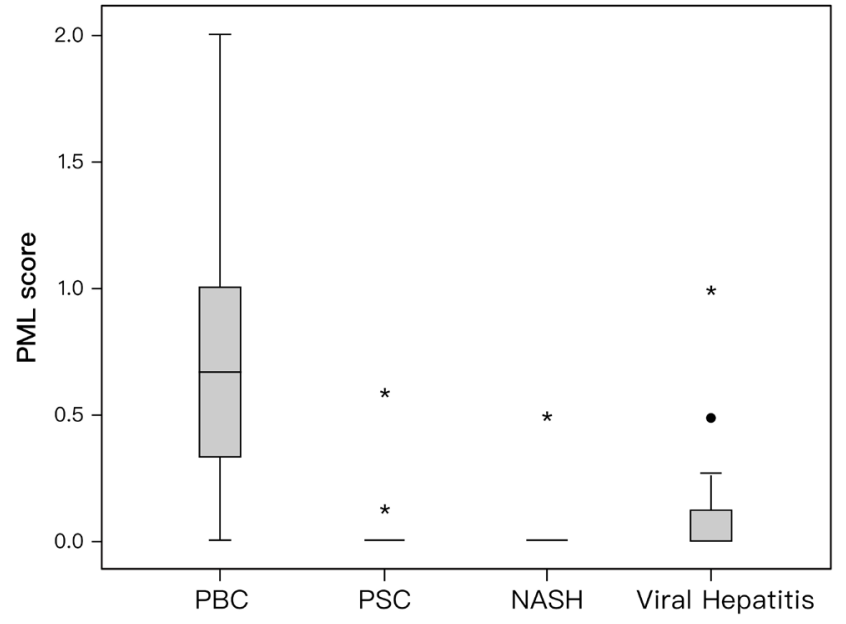

Figure 4: Box plot of promyelocytic leukemia (PML) score (median, quartiles, range) for primary biliary cholangitis (PBC), primary sclerosing cholangitis (PSC), non-alcoholic steatohepatitis (NASH) and patients with viral hepatitis. The PML score was significantly higher in PBC patients than in any other disease group $\left(P<0.0001\right.$ for each comparison). ${ }^{*}$ Defines extreme cases (cases with values more than 3 box lengths from the upper or lower edge of the box). $\bullet$ Defines outliers (cases with values between 1.5 and 3 box lengths from the upper or lower edge of the box). The box length is the interquartile range.

The ROC for PML-score is shown in Figure 5. The AUC was 0.917 (95\%CI: 0.84-0.99). The sensitivity and specificity for the histological diagnosis of $\mathrm{PBC}$ at the cut-off point of PML-score 0.18 was $84.6 \%$ and $89.7 \%$, respectively (Table 2). Using this cut-off point, patients were divided into two groups: those who were "positive" (PML-score > 0.18) and those who were "negative" (PMLscore $<0.18)$. Accordingly, 84.6\% (22/26) PBC patients were "positive" compared to only $5 \%(1 / 20)$ of PSC patients, $9.1 \%(1 / 11)$ of NASH, $13.5 \%(5 / 37)$ of those with chronic HBV and HCV and none of healthy $(P<$ 0.001 for each comparison, Figure 6).

In addition, ANA by IIFL were positive in 13/26 (50\%) PBC, 19/20 (95\%) PSC, 7/11 (63.6\%) NASH, 16/37 $(43 \%)$ patients with chronic viral hepatitis and in none of the healthy patients. However, MND-pattern on HEp2 cells was detected in 4/13 (30\%) ANA-positive with PBC, $2 / 7(28.5 \%)$ with NASH, $1 / 16(6 \%)$ with chronic viral hepatitis and in none of the PSC patients. Of note, all the 4 patients with PBC and MND-pattern on HEp2 had PML-score $>0.18$ on liver histology, while the 3 patients of the control groups with MND-pattern had "negative" PML-score. Furthermore, 4/26 (15.4\%) PBC patients were positive for anti-PML antibodies by immunoblot (none of the disease and healthy controls was positive), while 5/26 (19\%) PBC patients were antisp100 positive (only 1 patient with NASH was anti-sp100 positive). Of those 4 and 5 PBC patients, 1 patient who was positive for both autoantibodies (anti-PML and anti- 
Papamichalis et al.: Promyelocytic leukemia antigen expression: A histological marker for primary biliary cholangitis diagnosis?

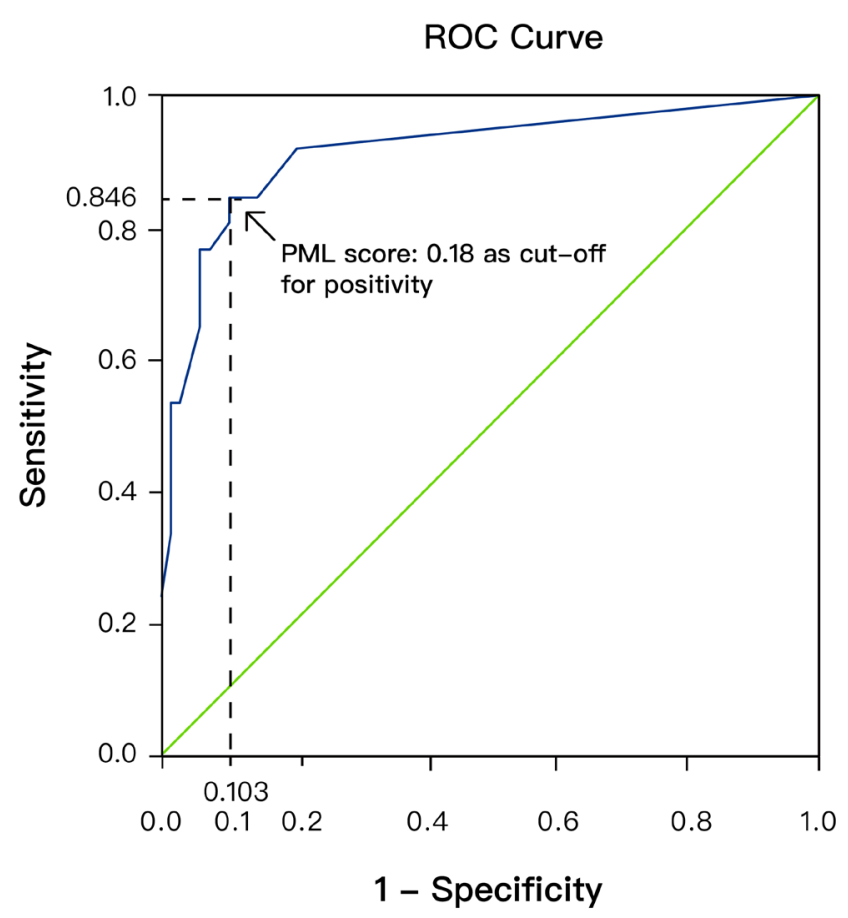

Figure 5: ROC curve for promyelocytic leukemia (PML) score. AUC: 0.917 (95\%Cl: 0.84-0.99). Sensitivity and specificity for the diagnosis of primary biliary cholangitis (PBC) at cut-off point 0.18 : $84.6 \%$ and $89.7 \%$, respectively.

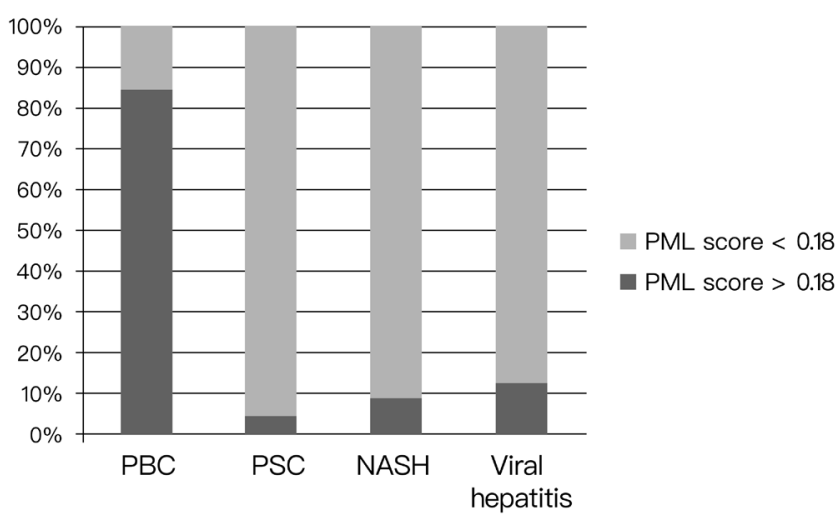

Figure 6: Immunoreactivity for promyelocytic leukemia (PML) expressed as PML score in different liver disease groups (cut-off point: 0.18 ). PBC: primary biliary cholangitis; PSC: primary sclerosing cholangitis; NASH: non-alcoholic steatohepatitis.

sp100), also had MND-pattern on HEp2. Finally, all the $4 \mathrm{PBC} /$ anti-PML positive and $4 \mathrm{PBC} /$ anti-sp100 positive patients by immunoblot had PML-score $>0.18$, while 1 $\mathrm{PBC} /$ anti-sp100 positive and the $1 \mathrm{NASH} /$ anti-sp100 positive patient did not show PML immunostaining on liver biopsy.

Although the number of PBC patients with PML-score $<0.18$ was limited $(n=4)$, we compared these patients with the rest PBC patients with PML-score $>0.18(n=$ 22). All the 4 PML-score "negative" patients had an early stage disease on liver biopsy (1 or 2 according to Ludwing's
Table 2: Sensitivity and specificity of the PML score

Patients with liver diseases Sensitivity Specificity

$(N=94)$

Positive/n (\%) Negative/N-n (\%)

$\mathrm{PBC}(n=26)$

22/26 (84.6) 61/68 (89.7)

PSC $(n=20)$

$1 / 20(5)$

$46 / 74(62.2)$

$\mathrm{NASH}(n=11)$

$1 / 11(9.1)$

$55 / 83(66.3)$

Viral Hepatitis $(n=37)$

$5 / 37(13.5)$

$33 / 57(57.9)$

- Chronic HBV infection

$4 / 25(16)$

$44 / 69(63.8)$

$(n=25)$

- Chronic HCV infection

$1 / 12(8.3)$

$54 / 82(65.9)$

$(n=12)$

PML: promyelocytic leukemia protein; PBC: primary biliary cholangitis; PSC: primary sclerosing cholangitis; NASH: non-alcoholic steatohepatitis; HBV: hepatitis B virus; HCV: hepatitis C virus. $N=$ the total number of patients; $n=$ the number of patients in each specific liver disease.

classification), although this did not reach statistical significance when compared with the PML-score "positive" PBC patients. In addition, PBC patients with "positive" PML-score had higher TBil levels than the PML-score "negative" patients $(0.65$ [0.4-1.59) vs. $0.4(0.35-0.7] ; P=$ 0.03). The GLOBE- and Mayo-risk scores as well as Paris II criteria of response to treatment did not differ between the two groups (data not shown).

Finally, when the PML "positive" and "negative" patients were taken into account irrespective of the kind of the underlying liver disease, the PML "positive" patients were older $(P=0.007)$, more often females $(P<0.001)$ and had higher ALP $(P<0.001), \gamma$-GT $(P=0.001)$ and IgM levels $(P$ $<0.001)$ compared to the PML "negative" patients (Table 3). Of interest, PML positive immunostaining in patients with chronic $\mathrm{HBV}$ and $\mathrm{HCV}$ was associated with a previous history of interferon therapy $(P=0.06)$. Indeed, all 5 out of 37 patients from the viral hepatitis group who tested "positive" (PML-score $>0.18$ ) had received interferon therapy (5 out of 19 patients who received interferon alpha-based treatment; $26.3 \%$ ).

\section{DISCUSSION}

In this study, we demonstrated for the first time in the English literature, a significantly higher frequency of immunohistochemical PML detection in PBC compared to other liver diseases, including PSC. Therefore, the PML immunostaining could be considered as a provisional histological marker of PBC (Figures 1, 2).

To the best of our knowledge, so far, only one study presented as an abstract has investigated the immunohistochemical PML detection indicating that PML-expression correlates significantly with the PBC stage and activity while a protective effect of PML on hepatocellular cancer development in PBC was also proposed. ${ }^{[34]}$ However, no disease controls were included in that study. 
Papamichalis et al.: Promyelocytic leukemia antigen expression: A histological marker for primary biliary cholangitis diagnosis?

\begin{tabular}{|c|c|c|c|}
\hline Patients characteristics & $\begin{array}{l}\text { PML score }>0.18 \\
(n=29)\end{array}$ & $\begin{array}{l}\text { PML score }<0.18 \\
(n=65)\end{array}$ & $P$ \\
\hline Age (years) & $53(32-73)$ & $45(16-76)$ & 0.007 \\
\hline \multicolumn{4}{|l|}{ Sex } \\
\hline $\begin{array}{l}\text { Male (\%) } \\
\text { Female (\%) }\end{array}$ & $\begin{array}{l}6(20.7) \\
23(79.3)\end{array}$ & $\begin{array}{l}44(67.7) \\
21(32.3)\end{array}$ & $<0.001$ \\
\hline AST (U/L, ULN:40) & $38(12-204)$ & $37(16-1286)$ & NS \\
\hline ALT (U/L, ULN: 40) & $46(9-324)$ & $54(9-1251)$ & NS \\
\hline ALP (U/L, ULN: 104) & $129(41-677)$ & $73(34-647)$ & $<0.001$ \\
\hline$\gamma$-GT (U/L, ULN: 40) & $87(22-883)$ & $38(7-874)$ & 0.001 \\
\hline $\lg M(\mathrm{mg} / \mathrm{dL})$ & $247(35.2-834)$ & $105(37-378.2)$ & $<0.001$ \\
\hline TBil (mg/dL, ULN: 1) & $0.7(0.35-1.6)$ & $0.7(0.4-4.8)$ & NS \\
\hline Albumin $(\mathrm{g} / \mathrm{dL})$ & $4.2(3.18-5)$ & $4.3(3.2-5.5)$ & NS \\
\hline Cirrhosis (yes/no) & $3 / 26$ & $7 / 58$ & NS \\
\hline
\end{tabular}

Data are expressed as median (range). NS: not statistically significant; PML: promyelocytic leukemia protein; AST: aspartate aminotransferase; ALT: alanine aminotransferase; $\gamma$-GT: gamma-glutamyltranspeptidase; ALP: alkaline phosphatse; TBil: total bilirubin.

Our particular method of quantification, in fact semiquantification, was selected for two reasons. First, in order to obtain a simplified PML-score to be used in different comparisons with other demographic, clinical and laboratory parameters of the patients and second, in order to represent accurately the histopathological impression gained after the thorough evaluation of the slides. During this evaluation, the pathologists involved in the study observed that in all the PBC cases, an intense staining of most nuclei in all the interlobular bile ducts was present. This picture was notable using a low power objective lens of the microscope and it was considered a distinct feature of PBC cases and a feature distinguishing these cases from all the other cases of the study. This observation provided the rational for duplicating the PML-score in these cases. Unfortunately, other methods of quantification for example the usage of an appropriate software in order to measure, and not simply estimate, the extent and the intensity of immunoreactivity along with the pertinent equipment were not available.

Regarding the rational of expressing the number of "immunostained" interlobular ducts in relation to the number of portal tracts, the following comments can be made. We refer to interlobular bile ducts and not the small, peripherally placed ductules. In the presence of complexity created by different factors, such as, the nature of the material, the focally increased intensity of inflammation, the need to compare and evaluate concurrently different levels/sections, including the $\mathrm{H}+\mathrm{E}$ slides, a less variable denominator was selected such as the number of portal tracts included in the biopsy. In our opinion, this approach does not affect the basic findings and it should also be noted that in some publications, ductopenia was also expressed as the quotient of bile duct number to the total portal tract number.
Interestingly, the PML tissue immunostaining showed an excellent correlation with the presence of serum antiPML autoantibodies detected by immunoblot, which uses purified PML antigen as substrate. However, although $84.6 \%$ of liver biopsies from PBC patients were stained positive for PML, only 4/26 of these patients tested positive for anti-PML antibodies by immunoblot in serum samples. Other studies in PBC sera have reported different detection rates of anti-PML (range: 8.7\%-42.3\%) when line immunoassay was used, ${ }^{[35-38]}$ while a prevalence of $19 \%-41.2 \%$ has been recorded when IIFL, combination of immunoprecipitation and IIFL or immunoblotting were used. ${ }^{[39,40]}$. However, the detection of an antigen on the target tissue significantly more frequently than the respective antibody in serum, could suggest its direct implication in disease pathogenesis and this might be of utmost importance. In addition, the immunohistochemical PML detection on liver PBC specimens seem to carry high specificity. This finding is in parallel with studies in serum, which highlighted the high specificity of anti-PML autoantibodies for PBC diagnosis. ${ }^{[41]}$

The correlation of immunohistochemical PML detection with older age and increased ALP, $\gamma$-GT, Tbil and IgM levels is concordant with other studies, which correlate the presence of anti-PML antibodies in serum samples of patients with more advanced liver disease. ${ }^{[3,4,34,38]}$ Indeed, a recent study has shown that serum samples with double reactivity against PML and sp100 antigens characterize patients with more advanced liver disease even though, this specific subgroup in that study was relatively small $(n=$ 10) to draw general conclusions. ${ }^{[38]}$ On the other hand, our finding regarding the association of immunohistochemical PML detection with previous administration of interferon in the group of viral hepatitis patients is in line with the fact that interferon is one of the known factors that can induce the overexpression of PML. ${ }^{[13,42]}$ Therefore, a previous 
history of interferon therapy should be cautiously ruled out when a positive PML staining is detected.

Of note in acute PML $\mathrm{t}(15 ; 17)$, the most common chromosomal translocation between PML and retinoic acid receptor a (RARa) genes is responsible for the production of the fusion protein PML-RARa, which is strictly connected with disease pathogenesis. ${ }^{[43]}$ In this context, the detection of PML by immunohistochemical staining on bone marrow and/or peripheral blood samples, serves as a sensitive and highly specific marker for acute PML diagnosis. ${ }^{[44,45]}$ Similarly, our results strongly suggest that there is an overexpression of PML in bile ducts of PBC (Figures 1,2). Interestingly, this finding seems to be unique and specific for $\mathrm{PBC}$ amongst other chronic liver diseases suggesting its potential use as an additional surrogate marker for PBC diagnosis. In acute PML, the administration of all-trans-retinoic acid (ATRA) or arsenic trioxide $\left(\mathrm{As}_{2} \mathrm{O}_{3}\right)$ leads to the correction of PML expression and restoration of PML-NBs structure. These two chemical substances are highly effective for acute PML with minimal toxicity for the patients. Identifying the molecular basis of PML overexpression in PBC, one could suggest the use of ATRA or $\mathrm{As}_{2} \mathrm{O}_{3}$ in an attempt to reverse PML overexpression in bile ducts, as a new specific therapeutic target for PBC patients not responding to ursodeoxycholic $\operatorname{acid}^{[1,2]}$

Last but not least, our study was able to underscore that PML overexpression on liver tissue could serve as a strong marker in an attempt to differentiate among the four subtypes of non-suppurative cholangitis. Such a differentiation that could not be achieved by morphological criteria alone, ${ }^{[14,21]}$ now can be accomplished by immunohistochemical staining. Indeed, the characteristic pattern of PML overexpression across PBC bile ducts along with its excellent specificity and sensitivity could assist in the differential diagnosis of the underlying clinical condition even when no other clinical or laboratory data is available.

In conclusion, our results demonstrated that immunohistochemical detection of PML on liver tissue may serve as a much more sensitive and specific marker for PBC compared to the respective detection of ANA PBCspecific antibodies in serum. This immunohistochemical PML detection on PBC liver tissues is almost comparable to the detection of AMA in serum. Therefore, PML detection by immunohistochemical assays could be used at least as a potential new biomarker for the differential diagnosis of problematic cases like AMA-negative PBC and small duct PSC variant, or when other concurrent liver diseases are suspected such as, AIH, NASH or viral hepatitis. ${ }^{[3,15]}$ In addition, the characteristic pattern of PML distribution on the bile ducts in PBC, suggests that it could be implicated in disease pathogenesis and/or that the antigen may play a unique role in disease progression. However, further multicenter studies are needed in order to clarify this novel observation, which in turn may lead in the future to potential new diagnostic and therapeutic implications in PBC.

\section{Source of Funding}

This research did not receive any specific grant from funding agencies in the public, commercial, or not-forprofit sectors.

\section{Conflicts of Interest}

All the authors have nothing to declare.

\section{Informed Consent}

Patients were not required to give informed consent to the study because the analysis used anonymous data that were obtained after each patient agreed to the standard diagnostic procedures and treatment of chronic liver diseases by oral consent.

\section{Author Contribution}

George N. Dalekos and George K. Koukoulis: Conception and design of the work, data interpretation, critical revision of the manuscript and final approval of the version to be published; George N. Dalekos: Diagnosis and treatment of patients; George K. Koukoulis: Histological diagnosis; Panagiotis Papamichalis, Nikolaos Gatselis and Kalliopi Zachou: Treatment of the patients, laboratory investigation, collection of the data, formal analysis, writing the first draft of the manuscript and final approval of the version to be published; Roidoula A. Papamichali and Maria Ioannou: Histological investigation, collection and interpretation of the data and final approval of the version to be published.

\section{REFERENCES}

1. EASL Clinical Practice Guidelines. The diagnosis and management of patients with primary biliary cholangitis. J Hepatol 2017;67:145-72.

2. Terziroli Beretta-Piccoli B, Mieli-Vergani G, Vergani D, Vierling JM, Adams D, Alpini G, et al. The challenges of primary biliary cholangitis: What is new and what needs to be done. J Autoimmun 2019; 105: 102328.

3. Dalekos GN, Gatselis NK. Variant and specific forms of autoimmune cholestatic liver diseases: A short review. Arch Immunol Ther Exp (Warsz) 2019; 67: 197-211.

4. Gatselis NK, Dalekos GN. Molecular diagnostic testing for primary biliary cholangitis. Expert Rev Mol Diagn 2016; 16: 1001-10.

5. Granito A, Muratori P, Quarneti C, Pappas G, Cicola R, Muratori L. Antinuclear antibodies as ancillary markers in primary biliary cirrhosis. Expert Rev Mol Diagn 2012; 12: 65-74. 
6. Norman GL, Yang CY, Ostendorff HP, Shums Z, Lim MJ, Wang J, et al. Anti-kelch-like 12 and anti-hexokinase 1: novel autoantibodies in primary biliary cirrhosis. Liver Int 2015; 35: 642-51.

7. Liu H, Norman GL, Shums Z, Worman HJ, Krawitt EL, Bizzaro N, et al. PBC Screen: an IgG/IgA dual isotype ELISA detecting multiple mitochondrial and nuclear autoantibodies specific for primary biliary cirrhosis. J Autoimmun 2010; 35: 436-42.

8. Granito A, Yang WH, Muratori L, Lim MJ, Nakajima A, Ferri S, et al. PML nuclear body component Sp140 is a novel autoantigen in primary biliary cirrhosis. Am J Gastroenterol 2010; 105: 125-31.

9. Nakamura $\mathrm{M}$, Kondo $\mathrm{H}$, Mori $\mathrm{T}$, Komori $\mathrm{A}$, Matsuyama $\mathrm{M}$, Ito $\mathrm{M}$, et al. Anti-gp210 and anti-centromere antibodies are different risk factors for the progression of primary biliary cirrhosis. Hepatology 2007; 45: 118-27.

10. Hu CJ, Song G, Huang W, Liu GZ, Deng CW, Zeng HP, et al. Identification of new autoantigens for primary biliary cirrhosis using human proteome microarrays. Mol Cell Proteomics 2012; 11: 669-80.

11. Rigopoulou EI, Davies ET, Pares A, Zachou K, Liaskos C, Bogdanos DP, et al. Prevalence and clinical significance of isotype specific antinuclear antibodies in primary biliary cirrhosis. Gut 2005; 54: 528-32.

12. Sternsdorf T, Guldner HH, Szostecki C, Grotzinger T, Will H. Two nuclear dot-associated proteins, PML and Sp100, are often co-autoimmunogenic in patients with primary biliary cirrhosis. Scand J Immunol 1995; 42: $257-68$.

13. Grotzinger T, Sternsdorf T, Jensen K, Will H. Interferon-modulated expression of genes encoding the nuclear-dot-associated proteins Sp100 and promyelocytic leukemia protein (PML). Eur J Biochem 1996; 238 : 554-60.

14. Ozaslan E, Efe C, Gokbulut Ozaslan N. The diagnosis of antimitochondrial antibody-negative primary biliary cholangitis. Clin Res Hepatol Gastroenterol 2016; 40: 553-561.

15. Dalekos GN, Lygoura V, Gatselis NK. Variants of primary biliary cholangitis: An updated mini review. J Autoimmune Dis Rheumatol 2018; 6: 21-8.

16. Ludwig J, Dickson ER, McDonald GS. Staging of chronic nonsuppurative destructive cholangitis (syndrome of primary biliary cirrhosis). Virchows Arch A Pathol Anat Histol 1978; 379: 103-12.

17. Scheuer PJ. Primary biliary cirrhosis: diagnosis, pathology and pathogenesis. Postgrad Med J 1983; 59(Suppl 4): 106-15.

18. Nakanuma Y, Zen Y, Harada K, Sasaki M, Nonomura A, Uehara T, et al. Application of a new histological staging and grading system for primary biliary cirrhosis to liver biopsy specimens: Interobserver agreement. Pathol Int 2010; 60: 167-74.

19. Harada K, Hsu M, Ikeda H, Zeniya M, Nakanuma Y. Application and validation of a new histologic staging and grading system for primary biliary cirrhosis. J Clin Gastroenterol 2013; 47: 174-81.

20. Kakuda Y, Harada K, Sawada-Kitamura S, Ikeda H, Sato Y, Sasaki M, et al. Evaluation of a new histologic staging and grading system for primary biliary cirrhosis in comparison with classical systems. Hum Pathol 2013; 44: 1107-17.

21. Ludwig J, Czaja AJ, Dickson ER, LaRusso NF, Wiesner RH. Manifestations of nonsuppurative cholangitis in chronic hepatobiliary diseases: morphologic spectrum, clinical correlations and terminology. Liver 1984; 4: 105-16.

22. Chapman MH, Thorburn D, Hirschfield GM, Webster GGJ, Rushbrook SM, Alexander G, et al. British Society of Gastroenterology and UK-PSC guidelines for the diagnosis and management of primary sclerosing cholangitis. Gut 2019; 68: 1356-78.

23. EASL 2017 Clinical Practice Guidelines on the management of hepatitis B virus infection. J Hepatol 2017; 67: 370-98.

24. EASL Recommendations on treatment of hepatitis C 2018. J Hepatol 2018; 69: 461-511.

25. EASL - EASD - EASO Clinical Practice Guidelines for the management of non-alcoholic fatty liver disease. J Hepatol 2016; 64: 1388-402.

26. Chalasani N, Younossi Z, Lavine JE, Charlton M, Cusi K, Rinella M, et al. The diagnosis and management of nonalcoholic fatty liver disease: Practice guidance from the American Association for the Study of Liver Diseases. Hepatology 2018; 67: 328-57.

27. Dickson ER, Grambsch PM, Fleming TR, Fisher LD, Langworthy A. Prognosis in primary biliary cirrhosis: model for decision making. Hepatology 1989; 10: 1-7.

28. Lammers WJ, Hirschfield GM, Corpechot C, Nevens F, Lindor KD, Janssen HL, et al. Development and validation of a scoring system to predict outcomes of patients with primary biliary cirrhosis receiving ursodeoxycholic acid therapy. Gastroenterology 2015; 149: 1804-12.

29. Corpechot C, Chazouilleres O, Poupon R. Early primary biliary cirrhosis: biochemical response to treatment and prediction of long-term outcome. J Hepatol 2011; 55: 1361-7.

30. Rigopoulou EI, Zachou K, Gatselis NK, Papadamou G, Koukoulis GK, Dalekos GN. Primary biliary cirrhosis in HBV and HCV patients: Clinical characteristics and outcome. World J Hepatol 2013; 5: 577-83.

31. Zografos TA, Gatselis N, Zachou K, Liaskos C, Gabeta S, Koukoulis GK, et al. Primary biliary cirrhosis-specific autoantibodies in first degree relatives of Greek primary biliary cirrhosis patients. World J Gastroenterol 2012; 18: 4721-8.

32. Gatselis NK, Zachou K, Norman GL, Gabeta S, Papamichalis P, Koukoulis GK, et al. Clinical significance of the fluctuation of primary biliary cirrhosis-related autoantibodies during the course of the disease. Autoimmunity 2013; 46: 471-9.

33. Gabeta S, Norman GL, Liaskos C, Papamichalis PA, Zografos T, Garagounis A, et al. Diagnostic relevance and clinical significance of the new enhanced performance M2 (MIT3) ELISA for the detection of $\operatorname{IgA}$ and IgG antimitochondrial antibodies in primary biliary cirrhosis. J Clin Immunol 2007; 27: 378-87.

34. Hintemann C, Straub SK, Biesterfeld S, Galle PR, Erthle J, Gerken G, et al. Nuclear proteins in primary biliary cirrhosis as guardians against HCC development [Abstract]. Z Gastroenterol 2013; 51: 23.

35. Saito H, Takahashi A, Abe K, Okai K, Katsushima F, Monoe K, et al. Autoantibodies by line immunoassay in patients with primary biliary cirrhosis. Fukushima J Med Sci 2012; 58: 107-16.

36. Villalta D, Sorrentino MC, Girolami E, Tampoia M, Alessio MG, Brusca I, et al. Autoantibody profiling of patients with primary biliary cirrhosis using a multiplexed line-blot assay. Clin Chim Acta 2015; 438: 135-8.

37. Li BA, Liu J, Hou J, Tang J, Zhang J, Xu J, et al. Autoantibodies in Chinese patients with chronic hepatitis B: prevalence and clinical associations. World J Gastroenterol 2015; 21: 283-91.

38. Mytilinaiou MG, Meyer W, Scheper T, Rigopoulou EI, Probst C, Koutsoumpas AL, et al. Diagnostic and clinical utility of antibodies against the nuclear body promyelocytic leukaemia and Sp100 antigens in patients with primary biliary cirrhosis. Clin Chim Acta 2012; 413; 1211-6.

39. Zuchner D, Sternsdorf T, Szostecki C, Heathcote EJ, Cauch-Dudek $\mathrm{K}$, Will H. Prevalence, kinetics, and therapeutic modulation of autoantibodies against Sp100 and promyelocytic leukemia protein in a large cohort of patients with primary biliary cirrhosis. Hepatology 1997; 26: 1123-30.

40. Xiao H, Chen JW, Xie X, Liu YM, Li F. [Diagnostic significance of autoantibodies in patients with primary biliary cirrhosis]. Beijing Da Xue Xue Bao 2012; 44: 209-14

41. Szostecki C, Guldner HH, Will H. Autoantibodies against "nuclear dots" in primary biliary cirrhosis. Semin Liver Dis 1997; 17: 71-8.

42. Gambacorta M, Flenghi L, Fagioli M, Pileri S, Leoncini L, Bigerna B, et al. Heterogeneous nuclear expression of the promyelocytic leukemia (PML) protein in normal and neoplastic human tissues. Am J Pathol 1996; 149: 2023-35.

43. Falini B, Mason DY. Proteins encoded by genes involved in chromosomal alterations in lymphoma and leukemia: clinical value of their detection by immunocytochemistry. Blood 2002; 99: 409-26.

44. Falini B, Flenghi L, Fagioli M, Lo Coco F, Cordone I, Diverio D, A et al. Immunocytochemical diagnosis of acute promyelocytic leukemia (M3) 
Papamichalis et al.: Promyelocytic leukemia antigen expression: A histological marker for primary biliary cholangitis diagnosis?

with the monoclonal antibody PG-M3 (anti-PML). Blood 1997; 90: 4046-53.

45. Villamor N, Costa D, Aymerich M, Esteve J, Carrio A, Rozman M, et al. Rapid diagnosis of acute promyelocytic leukemia by analyzing the immunocytochemical pattern of the PML protein with the monoclonal antibody PG-M3. Am J Clin Pathol 2000; 114: 786-92.

How to cite this article: Papamichalis PA, Zachou K, Papamichali RA, loannou M, Gatselis NK, Dalekos GN, et al. Promyelocytic leukemia antigen expression: A histological marker for primary biliary cholangitis diagnosis? J Transl Intern Med 2021; 9: 43-51. 